\title{
Extracapsular cataract extraction to phacoemulsification: why and how?
}

\begin{abstract}
Purpose To determine what factors influence a decision to move from extracapsular cataract extraction to phacoemulsification as the cataract removal technique of first choice, and to investigate how the transition is managed. Method Questionnaire surveys were carried out in 1996 and 1997 of consultant ophthalmologists in the former Oxford Region.
\end{abstract}

Result 'Evidence of clinical benefit' was the strongest factor influencing a decision to change technique, but 'fear of becoming a dinosaur' and 'peer pressure' were also important factors. Beliefs about advantages of phacoemulsification were little changed with increasing experience, but personal contraindications to phacoemulsification diminished with time. Hard nuclei, mature cataracts and poor zonular support remained relatively strong contraindications to phacoemulsification by the second survey. Only $46 \%$ of 14 respondents had performed phacoemulsification under supervision before 'going solo', but $64 \%$ felt well or adequately prepared for their first solo phacoemulsification. Influence of non-clinical factors such as equipment availability on the choice of technique did not diminish significantly between surveys. Increasing experience of phacoemulsification appeared to reinforce beliefs about its advantages. Conclusions Respondents appear to have adopted phacoemulsification with careful preparation, and enthusiasm for the technique has been reinforced by increasing experience.

B. Gonglore

R. Smith

Stoke Mandeville Hospital Aylesbury, UK

Mr Richard Smith, FRCS FRCOphth Eye Department Stoke Mandeville Hospital NHS Trust

Mandeville Road Aylesbury HP21 8AL, UK

Tel: +44 (0)1296315033

Fax: +44 (0)1296315437
BIPIN GONGLORE, RICHARD SMITH

During the last few years a quiet revolution has been occurring in ophthalmology in the United Kingdom in the form of a change from extracapsular cataract extraction to phacoemulsification as the preferred technique of cataract removal. Although a subject of considerable discussion amongst ophthalmologists, the transition to phacoemulsification has attracted relatively little attention from the popular press or from healthcare purchasers in comparison with the introduction of minimal-access techniques in general surgery or gynaecology. The transition to phacoemulsification appears to have been made on the basis of individual professional judgement of surgeons and it would seem from casual observation that district general hospital units performing high volumes of cataract surgery have tended to adopt phacoemulsification earlier and more enthusiastically than teaching hospitals. The process of learning a new technique for a familiar operation can be a time of stress and insecurity, but also a highly valuable learning exercise for surgeons. This study was conducted to find out what factors are important in the decision to adopt phacoemulsification as the preferred technique for cataract removal, and how that change is managed.

\section{Method}

A postal questionnaire was sent to 25 consultant ophthalmic surgeons in the six ophthalmic inpatient units in the former Oxford Region during the spring of 1996. The questions addressed the following areas: factors influencing the decision to start learning phacoemulsification, beliefs about the benefits that have been claimed for phacoemulsification, numbers of phacoemulsifications performed, learning experiences, current contraindications to phacoemulsification, non-clinical factors influencing the decision to perform phacoemulsification in an individual case, and the anticipated maximum proportion of cataracts which would be removed by phacoemulsification. Comments were invited on the subject of whether transition from 
extracapsular cataract surgery to phacoemulsification should be an individual decision or whether there should be a coordinated transition within units. Individuals who responded to this questionnaire were asked to complete an abbreviated version of the same questionnaire in June 1997, with the exception of those who indicated that they had no plans to learn phacoemulsification.

\section{Results}

Sixteen consultants $(64 \%)$ completed the first questionnaire, but two replied to say that they had no plans to learn phacoemulsification and were therefore unable to answer the questions. Complete responses were received from 14 (56\%) consultants. Personal knowledge of individuals who did not respond indicated that this group consisted mainly of surgeons who were very experienced at phacoemulsification and those who had no immediate plans to learn the technique. As the questions related mainly to the process of learning phacoemulsification, the responses appear to be representative of the experiences of those in the transition from extracapsular cataract extraction to phacoemulsification as the preferred method of cataract extraction. The 14 consultants who completed the first questionnaire all completed the second questionnaire.

Questions 1, 3, 7, 8, 9 and 14 were asked only in the first questionnaire. In questions 2, 10, 11 and 12, data from 1996 and 1997 for each consultant was paired and shifts in the paired responses were analysed using the two-tailed Wilcoxon Signed Rank Test, a non-parametric test used for comparing medians for paired samples. The Wilcoxon coefficient, $T_{+}$, is the sum of the positive ranks of pairs with non-zero differences and is expressed as a probability of the observed difference being due to chance.
Question 1. To what extent have the following factors influenced your decision to start performing phacoemulsification: (a) evidence of clinical benefit, (b) pressure from advertising, (c) fear of becoming a

'dinosaur', (d) pressure from trainees, (e) peer pressure, (f) boredom with current methods of cataract extraction?

(Allowed responses: A lot, A little, Not at all.)

Results are displayed in Fig. 1.

Question 2. From your reading of relevant literature, first-hand experience, discussion with colleagues, etc., what is your view of the following statements about phacoemulsification compared with extracapsular cataract extraction: (a) Phacoemulsification gives faster rehabilitation, (b) Phacoemulsification gives a better final visual outcome, (c) Phacoemulsification results in fewer intraoperative complications, (d) Phacoemulsification results in fewer post-operative complications, (e) Phacoemulsification results in fewer post-operative clinic visits, (f) Phacoemulsification makes day-case surgery safer, (g) Phacoemulsification will eventually cost no more than extracapsular cataract extraction. (Allowed responses: Very positive, Positive, Ambivalent, Sceptical, Negative.)

Median responses in 1996 were 'Positive' to statements (a), (d), (e), (f), (g) and (h), 'Ambivalent' to statement (b) and 'Sceptical' to statement (c). The median response to statement (c) in 1997 was 'Ambivalent' ( $p=0.05$ ), but opinions on the other seven statements showed no significant change.

Question 3. In what year did you perform your first phacoemulsification?

Results are displayed in Fig. 2.

Question 4. Approximately how many cataracts have you removed by phacoemulsification in total?

Results are displayed in Fig. 3.

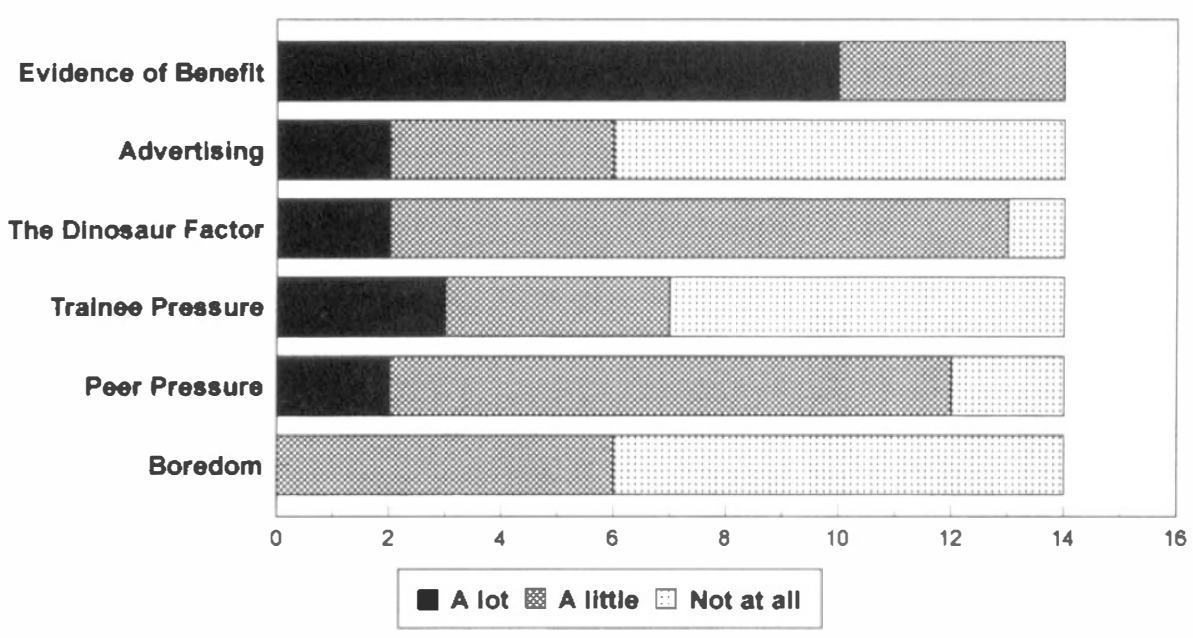

Fig. 1. Question 1. Factors influencing the decision to learn phacoemulsification. Fourteen respondents were asked to rate how strongly each factor had influenced their personal decision. 'The dinosaur factor' refers to a fear of being seen as out of date. 'Boredom' means boredom with previous technique of cataract extraction. 


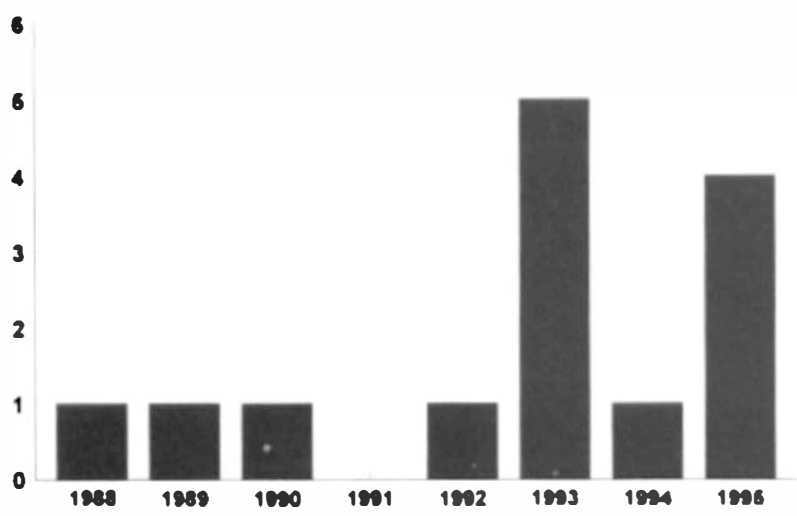

Fig. 2. Question 3. The year in which respondents performed their first phacoemulsification.

Question 5. Approximately how many

phacoemulsifications do you perform each month?

Results are displayed in Fig. 4.

Question 6. Approximately what percentage of cataract extractions do you currently perform by phacoemulsification?

Results are displayed in Fig. 5.

Question 7. Which of the following sources of instruction did you use prior to your first solo phacoemulsification: (a) textbooks/periodicals/videos, (b) courses/wet-labs, (c) attachment as a trainee under the supervision of an experienced consultant, (d) sabbatical period as a consultant? (More than one response allowed.)

Ten $(71 \%)$ had used textbooks, periodicals or videos as a source of instruction (in one case this was the only form of instruction). Thirteen (93\%) had attended courses or wet-labs. Six $(46 \%)$ had received instruction as a trainee under the supervision of an experienced consultant. No respondents had taken a sabbatical period as a consultant to learn phacoemulsification, though one $(7 \%)$ had learned the technique under the supervision of an experienced senior trainee.

Question 8. How well did these sources (collectively) prepare you for your first solo phacoemulsification?

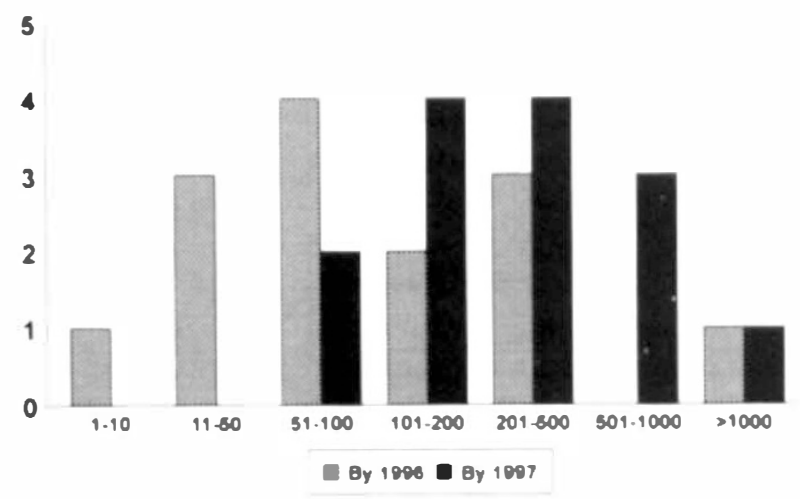

Fig. 3. Question 4. Fourteen respondents were asked in 1996 and again in 1997 to estimate the total number of phacoemulsifications performed to date. The $\mathrm{x}$-axis shows the number of phacoemulsifications.

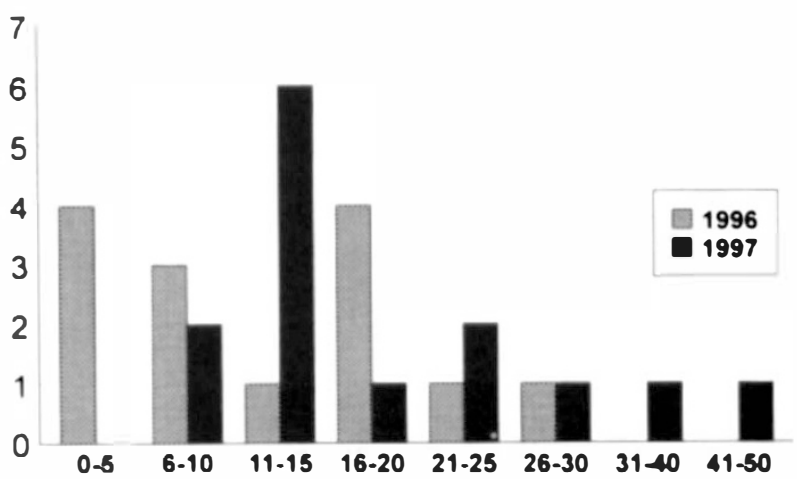

Fig. 4. Question 5. Fourteen respondents were asked in 1996 and again in 1997 to estimate the average number of phacoemulsifications they performed per month.

One respondent felt 'Well prepared'; 8 (57\%) felt 'Adequately prepared'; 4 (29\%) felt 'Barely adequately prepared' (including one who had attended courses and had received supervised instruction as a trainee); 1 felt 'Not at all prepared'.

Question 9. How easy or difficult did you find it to master the following steps of phacoemulsification technique: (a) self-sealing small incision, (b) capsulorhexis, (c) management of the nucleus, (d) irrigation/aspiration through a small incision, (e) insertion of foldable implant, (f) optimising machine settings? (Allowed responses: Nightmare, Difficult, Not too bad, Easy.)

Results are displayed in Fig. 6.

Question 10. To what extent do you currently regard the following situations as contraindications to phacoemulsification in your own practice: (a) nuclear sclerosis $3+$ or $4+$, (b) mature or hypermature cataract, (c) small pupil or posterior synechiae, (d) only seeing eye, (e) sub-optimal zonular support, (f) marked enophthalmos, ( $\mathrm{g}$ ) diabetes, $(\mathrm{h})$ glaucoma, (i) age less than 30 years, (j) corneal guttata, (k) local anaesthesia, (l) marked kyphoscoliosis? (Allowed responses: Always, Usually, Sometimes, Rarely, Never.)

Responses are shown in Table 1.

8

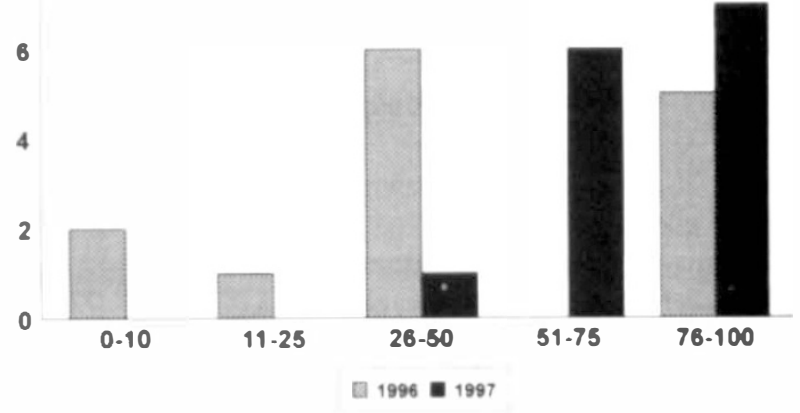

Fig. 5. Question 6. Fourteen respondents were asked in 1996 and again in 1997 to estimate the percentage of cataract extractions they performed by phacoemulsification. The $\mathrm{x}$-axis shows the percentage as phacoemulsification. 


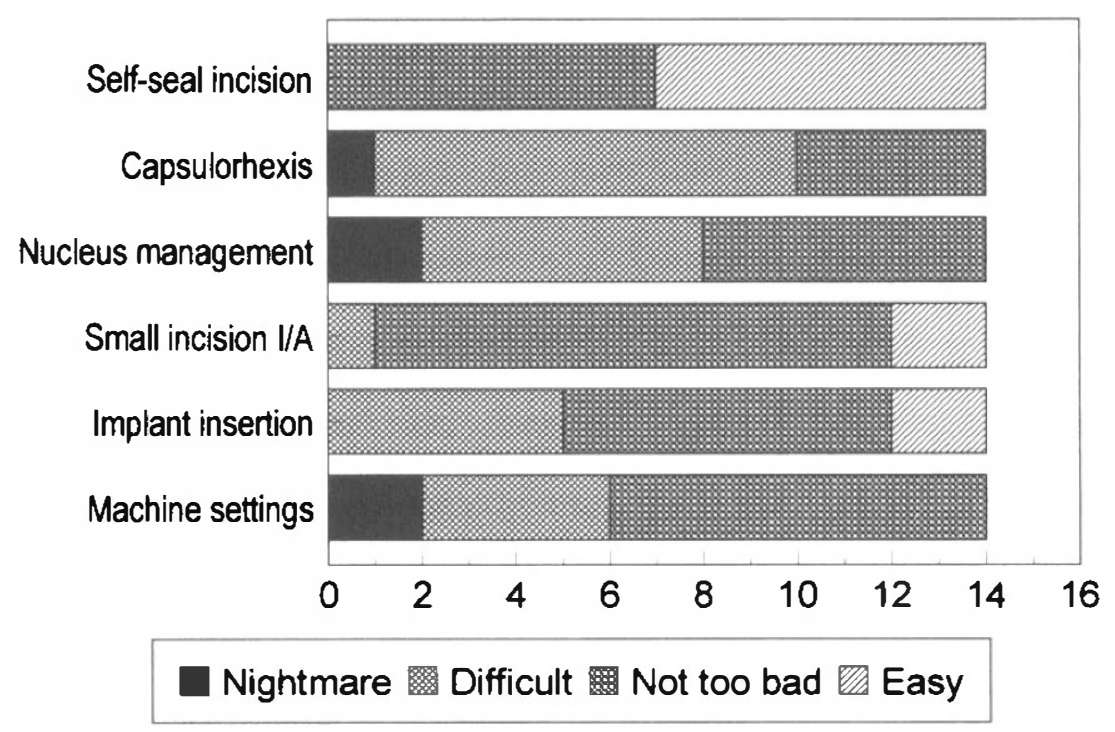

Fig. 6. Question 9. Fourteen respondents were asked to rate how difficult it was to learn various stages of phacoemulsification technique. I/A, means irrigation/aspiration.

Question 11. How often do the following factors influence your decision to perform phacoemulsification in an individual case: (a) availability of equipment, (b) pressure on theatre time, (c) results of your last phacoemulsification, (d) attitude of theatre staff to phacoemulsification? (Allowed responses: Often, Sometimes, Rarely, Never.)

Median responses in 1996 were (respectively): (a) 'Sometimes', (b) 'Sometimes', (c) 'Rarely', (d) 'Never'. There was no significant change when the question was asked again in 1997.

Question 12. To what extent do the following factors deter you from increasing the proportion of cataracts you remove by phacoemulsification: (a) uncertainty of clinical advantage over extracapsular cataract extraction, (b) having to rely on highly complicated equipment, (c) consequences of loss of nuclear material into the vitreous,

Table 1. Contraindications to phacoemulsification

\begin{tabular}{lcc}
\hline Clinical situation & $\begin{array}{c}\text { Median } \\
\text { response (1996) }\end{array}$ & $\begin{array}{c}\text { More or less } \\
\text { contraindicated in } \\
1997(p \text { value })\end{array}$ \\
\hline Nuclear sclerosis 3+ or 4+ & Usually & Less $(0.02)$ \\
Mature cataract & Usually & NS \\
Small pupil/synechiae & Usually & NS \\
Only seeing eye & Usually & Less $(0.01)$ \\
Poor zonular support & Always & NS \\
Marked enophthalmos & Sometimes & Less $(0.01)$ \\
Diabetes & Sometimes & Less $(0.02)$ \\
Glaucoma & Rarely & NS \\
Age <30 years & Rarely & NS \\
Corneal guttata & Sometimes & NS \\
Local anaesthesia & Never & NS \\
Orthopnoea & Rarely & NS \\
Marked kyphoscoliosis & Sometimes & NS \\
\hline NS, no & &
\end{tabular}

NS, no significant change. (d) uncertainty over long-term results, (e) limits of applicability of phacoemulsification to all types of cataract? (Allowed responses: A lot, A little, Not at all.)

Median responses in 1996 were: 'Not at all' for factors (a), (b) and (d) and 'A little' for factors (c) and (e). There was no significant change when the question was asked again in 1997.

Question 13. If equipment availability and theatre set-up allowed the use of phacoemulsification for every cataract extraction, what percentage of cataracts would you ultimately aim to remove by phacoemulsification?

Results are displayed in Fig. 7.

Question 14. Do you currently feel confident to supervise trainees performing phacoemulsification?

In 1996, 8 (57\%) respondents felt confident to supervise trainees learning phacoemulsification. By the second survey in 1997, 12 (86\%) felt confident to supervise the procedure.

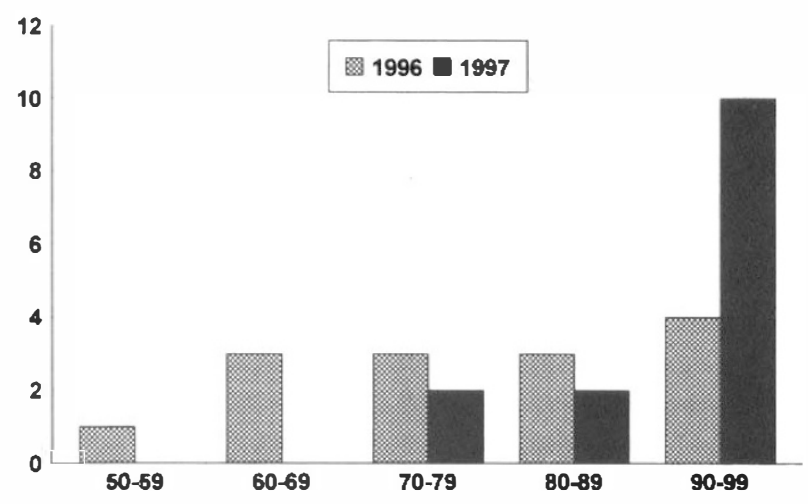

Fig. 7. Question 13. Fourteen respondents were asked in 1996 and again in 1997 what percentage of cataracts they would ideally aim to remove by phacoemulsification. The $\mathrm{x}$-axis shows the percentage. 
Question 15. Should any attempt be made as a unit to coordinate a transition from extracapsular cataract extraction to phacoemulsification as the method of first choice for cataract extraction?

The question was asked in 1996, when 6 (43\%) said 'Yes' and the remainder said ' $\mathrm{No}^{\prime}$ ' or were undecided.

\section{Discussion}

It was predictable that all respondents would cite evidence of clinical benefit' as one of the factors driving their decision to learn phacoemulsification. This does, however, raise the question of what constitutes evidence of clinical benefit.

Superiority of a small (i.e. $5 \mathrm{~mm}$ or less) self-sealing cataract incision over a conventional extracapsular cataract incision in terms of resistance to dehiscence ${ }^{1}$ and stability of post-operative refractive error ${ }^{2,3}$ has been clearly demonstrated. These factors, combined with a reduced need for sutures, potentially reduce the number of post-operative visits to outpatient clinics. A randomised controlled trial comparing outcomes following phacoemulsification and small-incision modified extracapsular cataract extraction is in progress at Moorfields Eye Hospital and the Oxford Eye Hospital but the results have not been published at the time of writing (P. Rosen, personal communication). Medline searches have failed to identify any prospective, randomised clinical trials comparing outcomes of phacoemulsification with conventional extracapsular cataract extraction. Recent studies of the benefits of cataract surgery in terms of improvements in visual performance and quality of life have demonstrated that the procedure is both safe and efficacious. ${ }^{4-15}$ However, it has been possible to draw only limited conclusions about the relative safety and efficacy of phacoemulsification and extracapsular cataract extraction. ${ }^{10,16}$ This may be due partly to the fact that a very large trial or meta-analysis would be necessary to demonstrate a clear benefit over an established procedure that is already very effective and very safe, but the search for evidence is not helped by the fact that many studies of the outcome of cataract surgery are impaired by fundamental flaws in study design and methodology. ${ }^{17}$ The NHS Centre for Reviews and Dissemination, which aims to inform NHS purchasing decisions, delivered the following verdict in a recent review of cataract management: ${ }^{18}$ 'The quality of research on cataract surgery is generally poor ... Most of the research in this area is based on case series which, because they do not use comparable control groups, may provide biased estimates of the impact of different treatment methods.' The review does not comment on the current trend towards phacoemulsification as the preferred method of cataract extraction beyond noting that phacoemulsification 'is generally believed to be more effective, but no completed RCTs directly comparing visual and health outcomes, complications or costs were... identified'.
What, then, informs this 'general belief' in the superiority of phacoemulsification over extracapsular cataract extraction in the minds of many ophthalmologists? There is certainly no hint in the answers given in this questionnaire that the transition from extracapsular cataract extraction to phacoemulsification had been embarked upon frivolously or on a whim. It is likely that the factors constituting 'evidence of clinical benefit' vary from individual to individual but include reading relevant published literature (even if scientifically flawed), observing at first hand the surgical practice and results of colleagues, holding informal discussions with peers, attending scientific meetings at which the subject is under discussion and audit of personal surgical results. Whilst it is absolutely right that such factors should inform an individual's decision to adopt a new surgical technique, one must question whether a nation-wide shift to that new technique is justified without the backing of more solid scientific evidence of its efficacy and safety compared with the established technique.

Peer pressure and the 'dinosaur factor' were the two next most important driving forces in a decision to start learning phacoemulsification. Few ophthalmologists can have attended the Royal College of Ophthalmologists annual congress or the Oxford Ophthalmic Congress in the last four years without having been asked by peers 'Are you doing phacoemulsification yet?' (or worse, 'Haven't you converted yet?') at least once. Even if the question was meant kindly, it can hardly fail to induce feelings of anxiety about being left behind in the march of progress.

What of the role of commercial interests in the trend towards phacoemulsification? Whilst only $6(43 \%)$ respondents in this survey felt that advertising had influenced their decision to start learning phacoemulsification, it would be hard to deny that ophthalmologists have been targeted by a very professional and powerful advertising campaign by the suppliers of phacoemulsification equipment. This is hardly surprising as an order to equip even a small department for phacoemulsification may be worth $£ 50000$ or more once the costs of the phacoemulsifier, handpieces and instrument sets are included. From the manufacturer's perspective, the window of opportunity for marketing is finite, as a department once equipped is unlikely to reinvest in phacoemulsification equipment for several years. The centrepiece of today's major ophthalmic meeting is not the auditorium or the poster hall but the trade exhibition, where eye-catching displays of equipment, hospitality areas and multimedia presentations all vie for attention. In a gadget-oriented and (still) male-dominated speciality, can the sophisticated design of phacoemulsification units and the unsubtle phallic symbolism of the phacoemulsification handpiece fail to have at least a subliminal effect on our decision-making?

Pressure from trainees who have done little or no phacoemulsification and want to learn the technique may not be too much of a problem (7 of 14 respondents said 
that pressure from trainees had influenced their decision to learn phacoemulsification), but it may be less easy to cope with a cohort of trainees whose previous experience of cataract surgery is entirely restricted to

phacoemulsification in a unit with limited experience of the technique amongst the consultants.

Respondents generally agreed with the statements about the benefits of phacoemulsification in question 2 . There was scepticism in 1996 that phacoemulsification might give a lower rate of intraoperative complications than extracapsular cataract extraction, but opinions had become more positive by 1997 and this was the only part of the question to show a statistically significant shift.

The 15 months between the first and second questionnaires has seen a substantial trend towards phacoemulsification as the preferred method of cataract extraction, as shown by Figs. 3-5. In 1996, 3 respondents with 3 or more years experience of phacoemulsification were still performing $40 \%$ or fewer cataract extractions by phacoemulsification, but by 1997 all 3 were performing phacoemulsification in $60 \%$ or more of cases. All respondents reported that the percentage of cataracts removed by phacoemulsification had increased between 1996 and 1997.

Five of the 14 respondents felt inadequately or barely adequately prepared for their first solo

phacoemulsification. Of these, 2 had had a period of supervised training in phacoemulsification before becoming a consultant, so this may owe as much to personal feelings as to the actual quality of training. The work pattern of most consultants makes it difficult in practice for them to operate under the supervision of consultant colleagues, as their immediate colleagues often have fixed commitments elsewhere when help is needed. In some units, there may be senior trainees with sufficient experience to fulfil this role. One factor that makes the learning curve of phacoemulsification less daunting than it might be is the fact that a number of the essential stages of the procedure can be performed as part of an extracapsular cataract extraction. Nevertheless, there is still a substantial gap to bridge between what can be learned from the wet-lab or modifications of extracapsular technique and the first 'live' phacoemulsification. Of the stages of the phacoemulsification technique, continuous circular capsulorhexis was the most difficult to master, followed by management of the nucleus and optimisation of machine settings.

A good indicator of increasing proficiency and confidence with a new technique is a change in the clinical situations regarded as absolute or relative contraindications to its use. A statistically significant reduction in the level of contraindication by 1997 was seen for harder nuclei, only seeing eyes, diabetic eyes and enophthalmic eyes. Respondents remained wary of mature cataracts, suspect zonules and poorly dilating pupils.

The decision to perform phacoemulsification rather than extracapsular cataract extraction in an individual case may be influenced by non-medical factors, especially where the surgeon is new to phacoemulsification. Availability of equipment remained an issue throughout the survey, probably because most units tend to buy instrument sets to satisfy current rather than future demand. The fact that 'pressure of theatre time' had no less influence on the choice of technique in 1997 than in 1996 suggests that the learning curve for phacoemulsification is quite prolonged. Respondents were largely undeterred by earlier setbacks or by resistance from theatre staff to accommodating the new technique. The results of question 12 suggest that respondents did not have significant anxieties about continuing the transition towards phacoemulsification as the technique of first choice and this is reflected in the increased optimism about the proportion of cataracts which could be removed by phacoemulsification from 1996 to 1997 (Fig. 7).

The overall impression gained from reading the answers and comments from the 14 consultants who participated in the study is that the transition from extracapsular cataract extraction to phacoemulsification has been approached with considerable caution and with careful preparation. Most commented that they had found the process of change stressful, but that they had not encountered as many problems as they had feared. Advice to colleagues about to make the transition highlighted the desirability of supervision of early cases by a more experienced colleague, or failing this, obtaining as much practice as possible in the wet-lab combined with rehearsal of technically difficult stages such as capsulorhexis on planned extracapsular cases.

This study has focused on the process of change from extracapsular cataract extraction to phacoemulsification as the preferred method of cataract extraction, rather than on outcomes. It would appear that the trend towards phacoemulsification nationally (and indeed across Europe and North America) is unstoppable, and therefore debate about the relative merits of the two techniques seems likely to wane over the next few years. The serious deficiencies in design and methodology of a large number of published studies of outcomes of cataract surgery is a cause for concern, and it is a pity that the opportunity to evaluate the two techniques prospectively with a large-scale randomised controlled trial earlier in this decade has been missed. Nevertheless, it seems likely that the change to small-incision cataract surgery will be viewed retrospectively as a positive one, although firm objective evidence of its benefits may have to be obtained using epidemiological methods other than the randomised controlled trial (for instance, a re-run of the National Cataract Survey ${ }^{19-21}$ ).

How long will it be before ophthalmologists are faced with another technical advance which requires mastery of a new set of practical skills for a commonly performed operation? Will the lessons learned in this transition be put to use in cushioning the impact of the next transition? We would dare to predict that in the future, ophthalmologists wishing to introduce new surgical techniques to their units will be expected to demonstrate effectiveness, cost-effectiveness and safety of the new 
technique with greater rigour than has been the case in the past. Purchasers, Clinical Risk Management teams and the lay public are all becoming more keenly interested in the processes that drive innovation in treatments, and following the 'Bristol incident' we cannot assume that surgeons' learning curves will remain a purely personal matter. Ophthalmologists must be ready to meet that challenge.

We wish to record our grateful thanks to Dr Brian Shine, FRCPath, for his help with statistical analysis, and to our consultant colleagues who participated in the study.

\section{References}

1. Ernest PH, Kiessling LA, Lavery KT. Relative strength of cataract incisions in cadaver eyes. J Cataract Refract Surg 1991;17:668-71.

2. Talamo JH, Stark WJ, Gottsch JD, et al. Natural history of corneal astigmatism after cataract surgery. J Cataract Refract Surg 1991;17:313-8.

3. Rainer G, Menapace R, Vass C, et al. Surgically induced astigmatism following a $4.0 \mathrm{~mm}$ sclerocorneal valve incision. J Cataract Refract Surg 1997;23:358-64.

4. Aylward G, Larkin D, Cooling R. Audit of cost and clinical outcome of cataract surgery. Health Trends 1993;25:126-9.

5. Klein BE, Klein R, Moss SE. Change in visual acuity associated with cataract surgery: The Beaver Dam Eye Study. Ophthalmology 1996;103:1727-31.

6. Abrahamsson M, Carlsson B, Tornquist M, Sterner B, Sjostrand J. Changes of visual function and visual ability in daily life following cataract surgery. Acta Ophthalmol Scand 1996;74:69-73.

7. Javitt JC, Street DA, Tielsch JM, et al. National outcomes of cataract extraction. Ophthalmology 1994;101:100-6.

8. Pfoff DS, Werner JS. Effect of cataract surgery on contrast sensitivity and glare in patients with $20 / 50$ or better Snellen acuity. J Cataract Refract Surg 1994;20:620-5.
9. Mangione CM, Phillips RS, Lawrence MG, et al. Improved visual function and attenuation of declines in health-related quality of life after cataract extraction. Arch Ophthalmol 1994;112:1419-25.

10. Powe NR, Schein OD, Gieser SC, et al. Synthesis of the literature on visual acuity and complications following cataract extraction with intraocular lens implantation. Cataract Patient Outcome Research Team. Arch Ophthalmol 1994;112:239-52.

11. Javitt JC, Brenner H, Curbow B, et al. Outcomes of cataract surgery: improvement in visual acuity in the first, second and both eyes. Arch Ophthalmol 1993;111:686-91.

12. Javitt JC, Steinberg EP, Sharkey $P$, et al. Cataract surgery in one eye or both: a billion dollar per year issue. Ophthalmology 1995;102:1583-92.

13. Desai P, Reidy A, Minassian DC, et al. Gains from cataract surgery: visual function and quality of life. $\mathrm{Br} \mathrm{J}$ Ophthalmol 1996;80:868-73.

14. Laidlaw A, Harrad R. Can second eye cataract surgery be justified? Eye 1993;7:680-6.

15. Lawrence D, Brogan C, Benjamin L, et al. The effectiveness of surgery: a study of a new patient-assessed cataract outcomes instrument. Buckinghamshire Health Board, 1995.

16. Schein OD, Steinberg EP, Javitt JC, et al. Variation in cataract surgery practice and clinical outcomes. Ophthalmology 1994;101:1142-52.

17. Powe NR, Tielsch JM, Schein OD, et al. Rigor of research methods in studies of the effectiveness and safety of cataract extraction with intraocular lens implantation. Arch Ophthalmol 1994;112:228-38.

18. NHS Centre for Reviews and Dissemination, University of York. Management of cataract. Effective Health Care 1996;[Feb] 2(3). Churchill Livingstone.

19. Courtney P. The national cataract surgery survey. I. Method and descriptive features. Eye 1991;6:487-92.

20. Desai P. The national cataract surgery survey. II. Clinical outcomes. Eye 1993;7:489-94.

21. Desai P. The national cataract surgery survey. III. Process features. Eye 1993;7:667-71. 